INPLASY

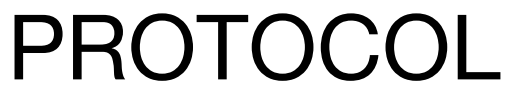

To cite: Shang et al. A metaanalysis of the efficacy and safety of Chinese herbal compound in the treatment of children with cough variant asthma. Inplasy protocol 202110087. doi: 10.37766/inplasy2021.1.0087

Received: 21 January 2021

Published: 22 January 2021

Corresponding author: Jiang Kai

jiangkaitianmei@sina.com

Author Affiliation:

Children's diagnosis and treatment center of Affiliated Hospital of Changchun University of traditional Chinese Medicine

Support: Funds allocated by the state.

Review Stage at time of this submission: Data analysis.

Conflicts of interest:

None.

\section{A meta-analysis of the efficacy and safety of Chinese herbal compound in the treatment of children with cough variant asthma}

Shang, GY1; Lin, SZ2 Wang, GL3; Qian, MJ4; Feng, XC5; Jiang, $K^{6}$.

Review question / Objective: Efficacy and safety of Chinese herbal compound in the treatment of children with cough variant asthma.

Condition being studied: The literatures of randomized controlled trials were searched from multiple databases at home and abroad. Revman5.4 and stata15.1 software were used for statistical analysis. Finally, a journal paper was completed.

Information sources: Electronic literature databases were searched by computer, including CNKI, Wanfang Data, VIP, CBM, PubMed and Cochrane Library. Trace the references of the required literature to improve the relevant retrieval literature. If the literature cannot be downloaded, conduct manual retrieval.

INPLASY registration number: This protocol was registered with the International Platform of Registered Systematic Review and Meta-Analysis Protocols (INPLASY) on 22 January 2021 and was last updated on 22 January 2021 (registration number INPLASY202110087).

\section{INTRODUCTION}

Review question / Objective: Efficacy and safety of Chinese herbal compound in the treatment of children with cough variant asthma.
Condition being studied: The literatures of randomized controlled trials were searched from multiple databases at home and abroad. Revman5.4 and stata15.1 software 
were used for statistical analysis. Finally, a journal paper was completed.

\section{METHODS}

Participant or population: Children with cough variant asthma.

Intervention: Chinese herbal compound.

\section{Comparator: Conventional Western Medicine.}

Study designs to be included: All the literatures were searched by two searchers and cross checked. The data were extracted by a unified data extraction table. The eligible literatures were selected according to the content of the search and the inclusion and exclusion criteria. If there was any disagreement between the two, they were agreed through internal discussion or decided after discussion with a third party judge.

Eligibility criteria: (1) All the subjects met the diagnostic criteria of cough variant asthma in children ]; age $(1<$ age < 18 years old) (2) randomized controlled clinical trials (RCTs) were included in the study, and the language was limited to Chinese or English, regardless of whether blind method or distribution concealment was adopted; (3) intervention measures: the trial group was treated with compound Chinese medicine, while the control group was treated with conventional western medicine. (4) OUTCOME MEASURES: the total effective rate, TCM syndrome score, recurrence rate and incidence of adverse reactions of the two groups after treatment.

Information sources: Electronic literature databases were searched by computer, including CNKI, Wanfang Data, VIP, CBM, PubMed and Cochrane Library. Trace the references of the required literature to improve the relevant retrieval literature. If the literature cannot be downloaded, conduct manual retrieval.
Main outcome(s): After treatment, the clinical total effective rate, TCM syndrome score, recurrence rate and incidence of adverse reactions of the two groups were compared.

Quality assessment / Risk of bias analysis: According to the Cochrane Handbook bias risk assessment tool, the evaluation content includes the following aspects: (1) generation of random sequence; (2) allocation concealment; (3) whether blind method is applied to the subjects and related personnel; (4) blind method is applied to the evaluation of effect indicators; (5) incomplete result data; (6) selective report of results; (7) other sources of bias.

Strategy of data synthesis: Revman $\mathbf{5 . 4}$ software provided by Cochrane Collaboration Network was used for metaanalysis and forest map was used to show the data. Relative risk (RR) was used to represent the difference between groups for binary variables, and mean difference (MD) was used for continuous variables.

Subgroup analysis: The experimental group was treated with compound Chinese medicine, and the control group was treated with conventional western medicine.

Sensibility analysis: Different statistical models or subgroups were selected and the possible sources of bias were explored.

Country(ies) involved: China.

Keywords: Traditional Chinese medicine compound; Children with cough variant asthma; Meta analysis; Randomized controlled trial.

Contributions of each author:

Author 1 - Shang GuangYuan.

Author 2 - Lin ShuangZhu.

Author 3 - Wang GuiLin.

Author 4 - Qian MeiJia.

Author 5 - Feng XiaoChun.

Author 6 - Jiang Kai. 